\title{
Functional Magnetic Resonance Imaging (fMRI) Activity in the Hippocampal Region during Recognition Memory
}

\author{
Craig E. L. Stark ${ }^{1}$ and Larry R. Squire ${ }^{1,2}$ \\ 1 University of California, San Diego, California 92093, and 2Veterans Affairs Medical Center, San Diego, California 92161
}

\begin{abstract}
Neuroimaging studies have often failed to observe activity in the hippocampal region during memory retrieval. Recently, two functional magnetic resonance imaging studies reported activity in the hippocampal region associated with recollective success. In both, participants studied pictures of objects and were given a recognition memory test with words that either did or did not name the studied objects. The recognition test was therefore cross-modal or associative in nature. These findings raise the question of what circumstances are required to observe activity in the hippocampal region during memory retrieval. Here, we
\end{abstract}

report that robust hippocampal activity for targets relative to foils occurred during retrieval in a recognition memory task when single words were used at both study and test, as well as when pictures of single nameable objects were used at both study and test. The hippocampal region is involved not just in overtly associative tasks but more broadly in the recollection of recently occurring facts and events.

Key words: recognition memory; fMRl; hippocampus; declarative memory; amnesia; associations
Lesion studies of humans and experimental animals have identified a system of medial temporal lobe (MTL) structures essential for the formation of declarative memory (memory for facts and events) (Milner et al., 1998). The contributions of the separate components of this system are not well understood. Recently, neuroimaging techniques have provided additional evidence for the importance of the MTL in learning and memory (for review, see Lepage et al., 1998; Schacter and Wagner, 1999) and have begun to examine the role of specific structures during encoding and retrieval. For example, during encoding, MTL activity is lateralized based on stimulus type. Activity is usually left unilateral during the encoding of words (Martin et al., 1997; Wagner et al., 1997; Kelly et al., 1998) (but see Fernandez et al., 1998) and bilateral during the learning of objects or pictures (Gabrieli et al., 1997; Martin et al., 1997; Brewer et al., 1998; Kelly et al., 1998). Furthermore, the level of activity in the MTL during learning is positively correlated with subsequent recognition memory accuracy (Wagner et al., 1997; Brewer et al., 1998; Fernandez et al., 1998).

Neuroimaging has been used less often to study memory retrieval, no doubt in part because of early failures to demonstrate activity in the MTL during retrieval (Grasby et al., 1993; Kapur et al., 1994; Shallice et al., 1994; Tulving et al., 1994; Buckner et al., 1995; Fletcher et al., 1995; Haxby et al., 1996). More recently, however, a number of studies have reported activity in the MTL during retrieval tasks (for review, see Lepage et al., 1998; Schacter and Wagner, 1999). Some of these studies contrasted activity during retrieval with activity during a perceptual baseline (a broad contrast that includes many cognitive functions, including the intention and effort to retrieve). Other studies contrasted the activity associated with studied target items with activity associated with unstudied foil items to identify brain regions associated with recollective success. Of the studies that compared targets with foils, most used positron emission tomography (PET) to observe activity in the MTL during retrieval (Nyberg et al., 1995; Fujii et al., 1997;

Received May 4, 2000; revised Aug. 2, 2000; accepted Aug. 2, 2000.

This research was supported by the Medical Research Service of the Department of Veterans Affairs, National Institute of Mental Health Grants MH24600 and MH12278, the National Alliance for Research in Schizophrenia and Depression, and the Metropolitan Life Foundation. We thank Shauna Stark, Jennifer Frascino, Joyce Zouzounis, and Cecelia Kemper for their assistance with data collection.

Correspondence should be addressed to Larry R. Squire, Veterans Affairs Medical Center, 116-A, 3350 La Jolla Village Drive, San Diego, CA 92161. E-mail: lsquire@ucsd.edu.

Copyright (C) 2000 Society for Neuroscience $0270-6474 / 00 / 207776-06 \$ 15.00 / 0$
Schacter et al., 1997). However, with PET, the limited spatial resolution $(1-2 \mathrm{~cm})$ makes it difficult to isolate the hippocampal region (the CA fields of the hippocampus, the dentate gyrus, and the subiculum) from adjacent structures in the parahippocampal gyrus.

Two studies have found bilateral activity in the hippocampal region at retrieval for targets versus foils using the higher resolution available with functional magnetic resonance imaging (fMRI) (Gabrieli et al., 1997; Stark and Squire, 2000). In both studies, participants studied pictures of objects and were tested with words that either did or did not name the objects. This task requires the formation or use of associations between objects and words. Whether such a cross-modal or associative task is required to observe hippocampal activity has broad theoretical implications for understanding the role of the hippocampus. Indeed, it has been suggested that the hippocampus is essential for forming and using associations and that simpler tasks of recognition memory can be supported by the adjacent cortex (Henke et al., 1997, 1999; Murray and Mishkin, 1998; Aggleton and Brown, 1999). We now report that robust hippocampal activity occurs during retrieval when single words are used at both study and test, as well as when single nameable objects are used at both study and test. Because there have been very few studies reporting hippocampal activity during recognition memory testing, we conducted our experiments independently at two different fMRI facilities to ensure the reliability of our results. The same results were obtained at both facilities.

\section{MATERIALS AND METHODS}

The participants were 9 male and 13 female right-handed volunteers $(5$ in each condition at site 1 and 6 in each condition at site 2; mean age, 26 years: range, 18-38 years) who gave informed consent before the study. Participants first viewed 80 items, twice each [duration, 2 sec; intertrial interval (ITI), $0.5 \mathrm{sec}$ ] outside the scanner with instructions to study the items for a later test. For half the participants $(n=11)$, the stimuli were line drawings of common nameable objects (Snodgrass and Vanderwart, 1980). For the other half $(n=11)$, the stimuli were words presented in capital letters that named the objects. Approximately $30 \mathrm{~min}$ after this study phase, 160 items ( 80 studied targets and 80 nonstudied foils) were presented on a screen located at the participant's feet (duration, $1.5 \mathrm{sec}$; ITI, $0.5 \mathrm{sec}$ ) while fMRI data were collected. Using their right hands, participants pressed one button on a response box to indicate that the item was studied (target) and another to indicate that it was not (foil). Which items served as targets and foils were counterbalanced across participants. A variant of the "boxcar" or blocked design was used in which test items were presented in eight blocks of 20 items each ( $40 \mathrm{sec} / \mathrm{block})$. Blocks alternated between primarily targets (T; 18 targets and 2 foils) and primarily foils (F; 18 foils and 2 targets). Additionally, at site 2, $40 \mathrm{sec}$ "rest" periods (R) were placed at the beginning and end of the recognition memory test (RTFT- 
FTFTFTFR). The recognition test was given twice to each participant $(\sim 3$ min between tests 1 and 2$)$. The two tests were identical, except that the order of items was different in each test.

Imaging parameters. At site 1 , imaging was performed on a General Electric (Milwaukee, WI) 1.5T Signa clinical MRI scanner fitted with high-performance local head gradient and radio-frequency coils (Wong et al., 1992a,b). Functional T2*-weighted images were acquired using an echoplanar, single-shot pulse sequence with a matrix size of $64 \times 64$, echo time (TE) of $40 \mathrm{msec}$, flip angle of $90^{\circ}$, and in-plane resolution of $3.75 \times$ $3.75 \mathrm{~mm}$. For each scanning run, 90 images were acquired for each of 21 sagittal $6 \mathrm{~mm}$ slices in an interleaved fashion with a repetition time (TR) of $3.6 \mathrm{sec}$. The first two images from each slice were discarded to assure that the MR signal had reached equilibrium on each slice. At site 2 , imaging was performed on a Siemens (Erlangen, Germany) 1.5T Vision clinical MRI scanner fitted with a large clinical "flex" coil and a bite bar. Functional $\mathrm{T} 2 *$-weighted images were acquired using an echoplanar, single-shot pulse sequence with a matrix size of $64 \times 64$, TE of $43 \mathrm{msec}$ flip angle of $90^{\circ}$, and in-plane resolution of $4 \times 4 \mathrm{~mm}$. For each scanning run, 164 images were acquired for each of 16 4-mm-thick slices aligned with the principal axis of the hippocampus in an interleaved fashion with a TR of $2.475 \mathrm{sec}$. In Figures 1-3, the approximate area covered by these slices at site 2 is indicated by a green box. The first four images from each slice were discarded to assure that the MR signal had reached equilibrium on each slice. At both MRI sites, a high-resolution MP-RAGE structural scan was acquired for anatomical localization.

Image analysis. Images from site 1 were first corrected for distortion attributable to field inhomogeneity (Reber et al., 1998). This capability was not available at site 2. At both sites, images were then co-registered through time using a two-dimensional (2D) registration algorithm (Cox, 1996). Each slice was spatially smoothed using a 2D (in-plane) Gaussian kernel; full width half-maximum $=$ two voxels. Within each run, voxels were eliminated if the signal magnitude changed $>8 \%$ between two samples or if the mean signal level was below a threshold defined by the inherent noise in the data acquisition. Such voxels (none were located in the hippocampal region) are likely to be contaminated by motion effects, venous effects, or exceptionally poor signal-to-noise ratios and cannot contain reliable data. Each participant's runs were then averaged and transformed (Collins et al., 1994; Cox, 1996) to conform to the atlas of Talairach and Tournoux (1988) using a nine-parameter transformation matrix with a final voxel size of $2.5 \mathrm{~mm}^{3}$. Data from all participants were then averaged.

Areas exhibiting activity selective for targets versus foils were identified by cross-correlating the time course of activity in each voxel against a set of seven time-shifted versions of an idealized reference function derived from the eight alternating blocks of targets and foils (Cox, 1996). An initial idealized reference function was first constructed to reflect the lag $(\sim 6 \mathrm{sec})$ between neural activity and hemodynamic response using a gamma function to model the response to each event. Six additional versions of this reference function were then constructed by shifting the idealized reference function both forward and backward in time in three 1 sec steps. By cross-correlating activity with multiple versions of the initial reference function, it is possible to allow for potential subject- and voxel-wise variations in the hemodynamic delay and for differences in the time of acquisition of each slice. Low-frequency noise was removed by including a third-order polynomial term in the cross-correlation procedure. The resulting statistical maps were then thresholded at a two-tailed $\alpha$ level of 0.01 (site 1, $r>0.326$; site 2 and the two sites combined, $r>0.265$ ). A clustering algorithm was then applied to remove voxels that were not part of a cluster of at least $125 \mathrm{~mm}^{3}$ of contiguous tissue (eight resampled $2.5 \mathrm{~mm}^{3}$ voxels).

Combining data from the two MRI facilities. The fMRI data from the two sites differed in their temporal resolution. At site 1, 88 samples of each voxel's activity (TR, $3.6 \mathrm{sec}$ ) were taken during each 5 min $17 \mathrm{sec}$ test. At site 2, 128 samples of each voxel's activity (TR, $2.475 \mathrm{sec}$ ) were taken during the same test along with 16 samples before and after the test began. To combine the data from the two sites, the fMRI data (averaged across participants) from each of the conditions were transformed as follows. First, the 88 samples of the activity of each voxel in the averaged data from site 1 (TR, $3.6 \mathrm{sec}$ ) were linearly resampled into 128 samples (TR, 2.4675 sec). The two 128 sample data sets from the two sites were then averaged, and the resulting data set was masked to include only voxels that contained reliable data in both data sets.

Region of interest analysis. The fMRI data from site 2 included $40 \mathrm{sec}$ rest
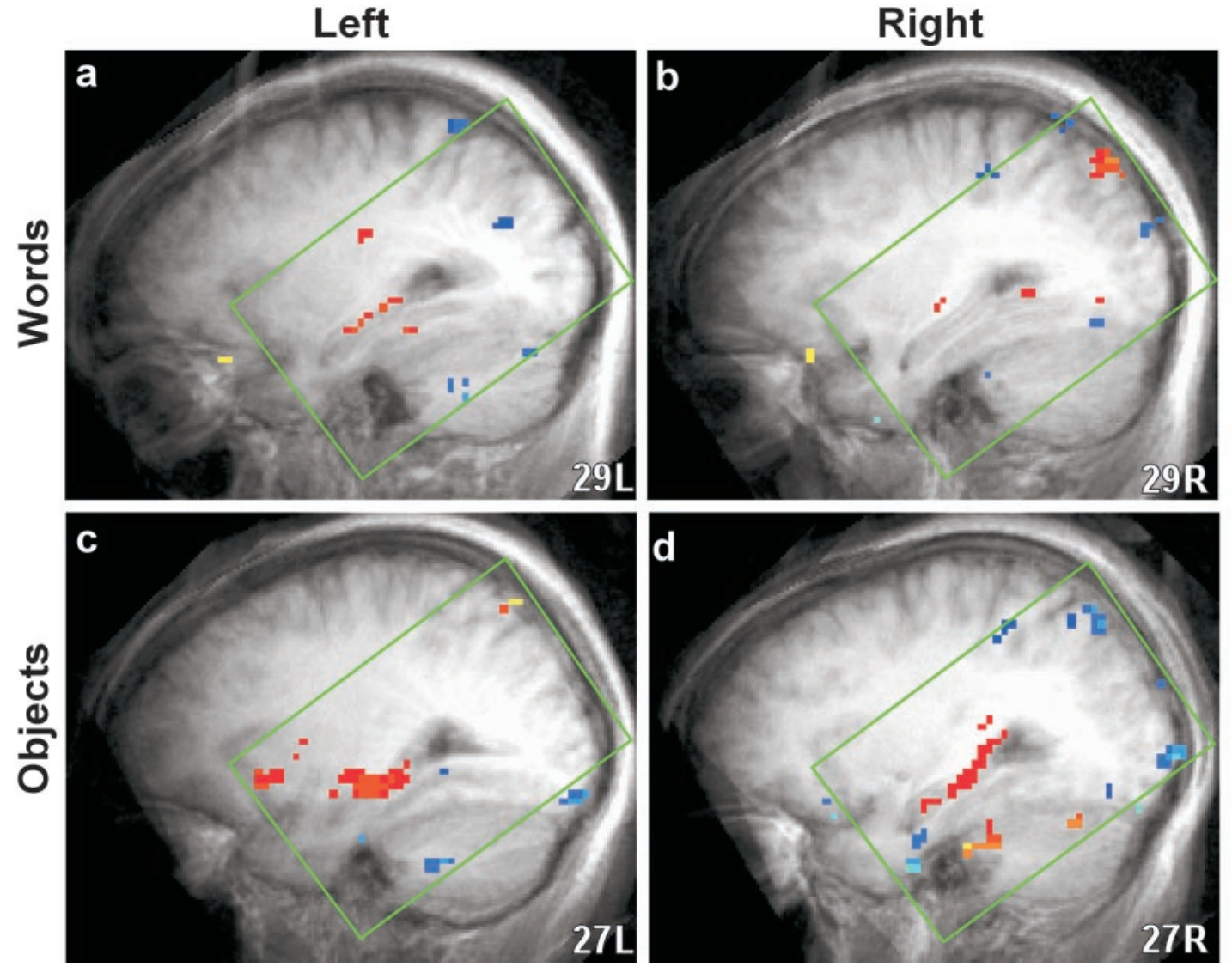

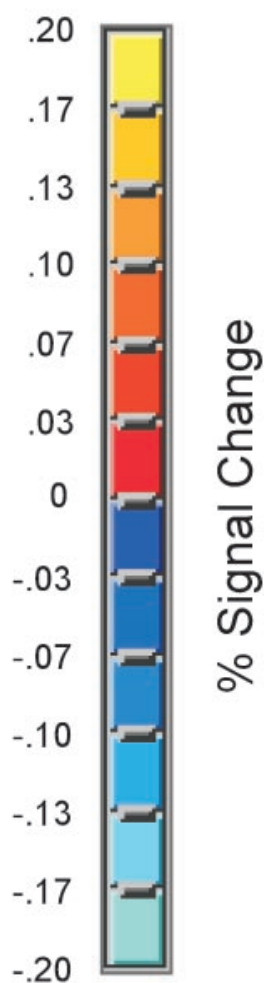

Figure 1. Activity in the left and right hippocampal regions. Shown are fMRI data from 11 participants who saw words at both study and test (Words) and from 11 different participants who saw pictures of nameable objects at both study and test (Objects), averaged across two MRI facilities and two recognition memory tests. Recognition memory accuracy was $80.2 \%$ correct for words and $89.9 \%$ correct for pictures of objects. Areas of significant fMRI signal change (targets vs foils) are shown in sagittal sections as color overlays on the averaged structural images [transformed to the atlas of Talairach and Tournoux (1988) at 29 left (L) and 29 right (R) (Words) and 27L and 27R (Objects)]. At site 1, whole-brain imaging was performed, but at site 2, imaging data were obtained from sixteen $4 \mathrm{~mm}$ slices aligned with the principle axis of the hippocampus. The green box indicates the area in which reliable data were available. When words were used at study and at test, activity was observed in the left $(a)$ but not in the right $(b)$ hippocampal region. When nameable objects were used at study and at test, activity was observed in both the left $(c)$ and the right $(d)$ hippocampal regions. 

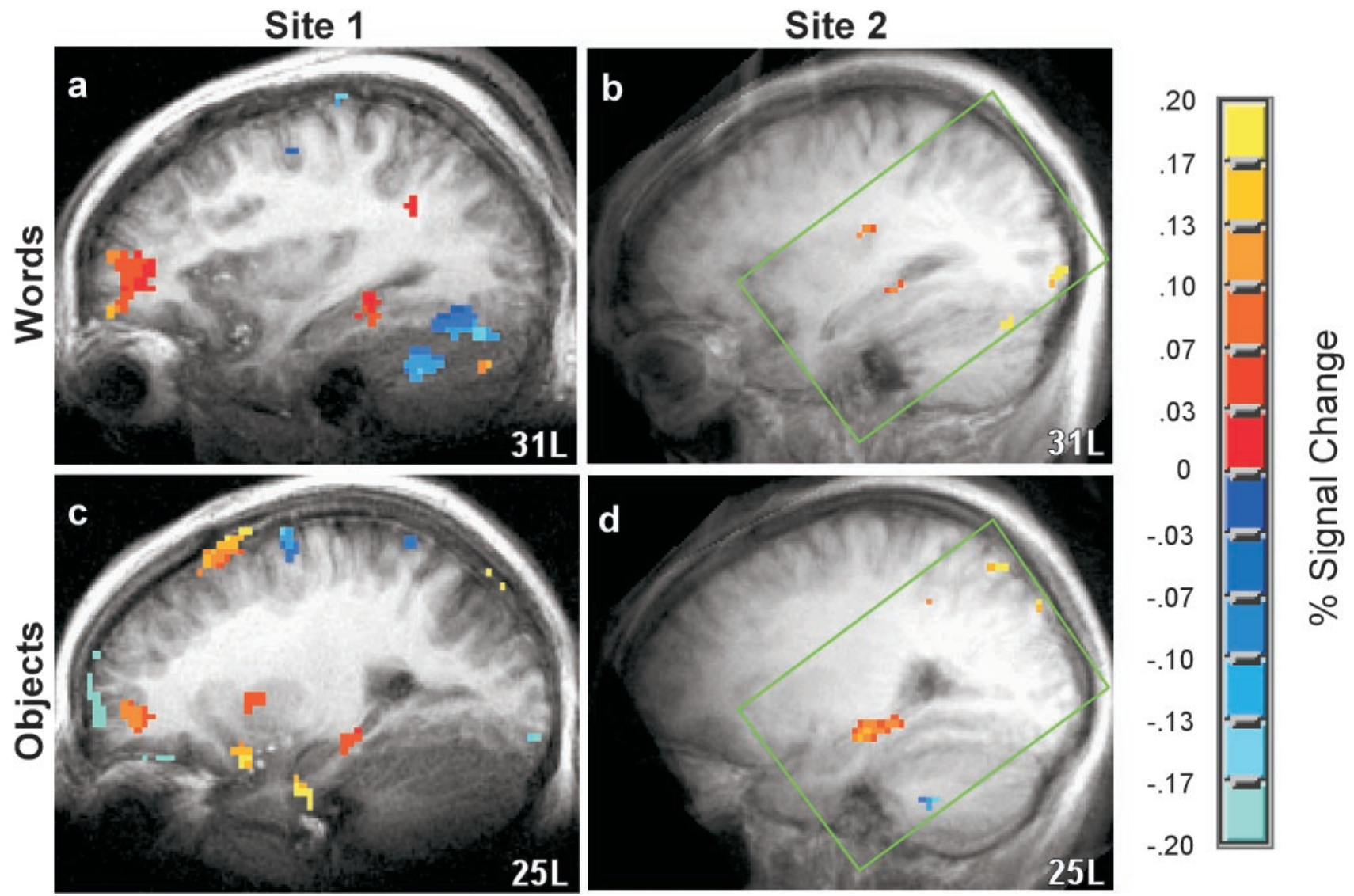

Figure 2. Hippocampal region activity: sites 1 and 2. fMRI data from participants in both the Words and Objects conditions (two recognition tests for each condition) were analyzed separately at each of the two MRI facilities to assess the reliability of the findings (five participants in each condition at Site 1 and six participants in each condition at Site 2). Areas of significant fMRI signal change (targets vs foils) in the left hippocampal region are shown in sagittal sections as color overlays on the averaged structural images [transformed to the atlas of Talairach and Tournoux (1988) at 31L and 25L]. The green box indicates the area in which reliable data were available from Site 2. The findings were similar at the two sites. When participants saw words at both study and test, activity was observed at test in the left hippocampal region $(a, b)$. When participants saw nameable objects at both study and test, activity was also observed at test in the left hippocampal region $(c, d)$. For objects, activity was also observed in the right hippocampal region at test (data not shown) but reached significance only at Site 2 .

periods immediately before and after each of the two recognition memory tests. Regions of interest (ROIs) were defined by the clusters of activity observed in the fMRI data, averaged across the two recognition memory tests. Within the left hippocampal region, an 8-voxel ROI was present when participants were shown words at both study and test, and a 90-voxel ROI was present when participants were shown objects at both study and test. The fMRI data from the voxels within each of these two ROIs were first averaged for tests 1 and 2 separately. Low-frequency drift within each ROI was then removed by a third-order polynomial fit, and each of the 160 samples $(2.475 \mathrm{sec} / \mathrm{sample})$ was classified as falling within one of the three task categories: rest, target, or foil. Because of the long rise and fall times associated with the blood oxygen level-dependent (BOLD) effect, samples that were acquired during a transition between blocks were discarded when at least $85 \%$ of the activity could not be assigned to one of the three task categories. The mean activity within the ROIs associated with the rest periods was then used as a baseline to calculate percent change associated with the presentation of targets and foils. It should be noted that although the rest period can provide a stable baseline across tests, it does not represent a measure of zero activity in the hippocampal region.

\section{RESULTS}

Using the statistical procedures described above, activation for targets versus foils was observed in the hippocampal region when either words or objects were being remembered (Fig. 1, combining across two MRI facilities and two successive recognition tests). Activation occurred in the left hippocampal region when participants saw words at both study and test (Fig. $1 a, b$ ), and activation occurred bilaterally when participants saw objects at both study and test (Fig. $1 c, d)$. Because the activation of the hippocampal region during retrieval has been infrequently reported, the same experiments were conducted at two different MRI sites to determine the reliability of the results. The results were strikingly consistent at the two different MRI sites (Fig. 2, Site 1, Site 2, combining across the two recognition tests that were given with words and across the two recognition tests that were given with objects). At each MRI facility, activity was observed in the left hippocampal region when either words or objects were being remembered, and the location of activity within the hippocampal region was virtually identical at the two facilities (Fig. 2, Words, posterior hippocampus; Objects, middle of the left hippocampus and the full anteroposterior extent of the right hippocampus). In the right hippocampal region (data not shown), activity was observed at both sites when objects were being remembered but reached significance only at site 2 .

We also examined activity in the hippocampal region separately during each of the two recognition memory tests (the same material was used in each test). Combining across the two MRI facilities, activation of the hippocampal region (targets vs foils) was observed (Fig. $3 a-d$ ) during the first of the two word recognition tests (Test 1) and during the second of two object recognition tests (Test 2). This same pattern of results was observed in the data from each of the MRI facilities as well (data not shown). Specifically, at each site, recognizing words (vs foils) activated the left hippocampal region during test 1 but not during test 2 . Furthermore, at each site, recognizing objects (vs foils) activated the hippocampal region bilaterally during test 2 but not during test 1 .

At site 2, fMRI data were also collected during $40 \mathrm{sec}$ rest periods immediately before and after each of the two recognition memory tests. Hippocampal activity during these rest periods was then used as a baseline against which to assess activity associated with both targets and foils (Fig. 3e,f). When participants saw words 
Test 1
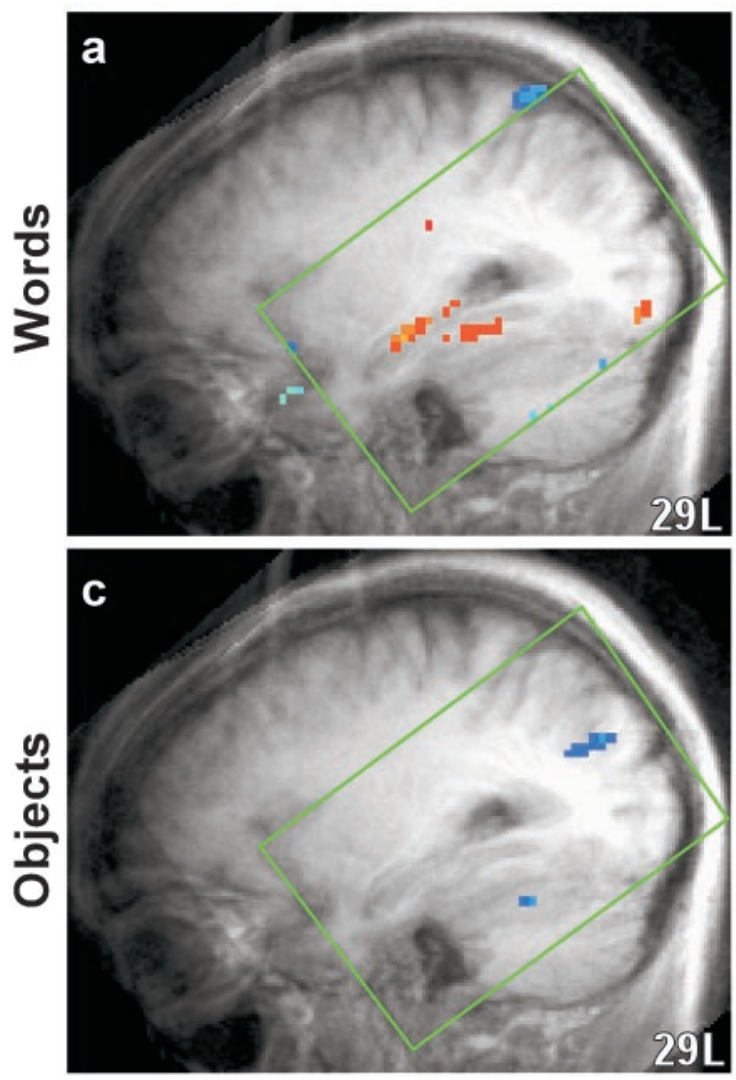

Test 2
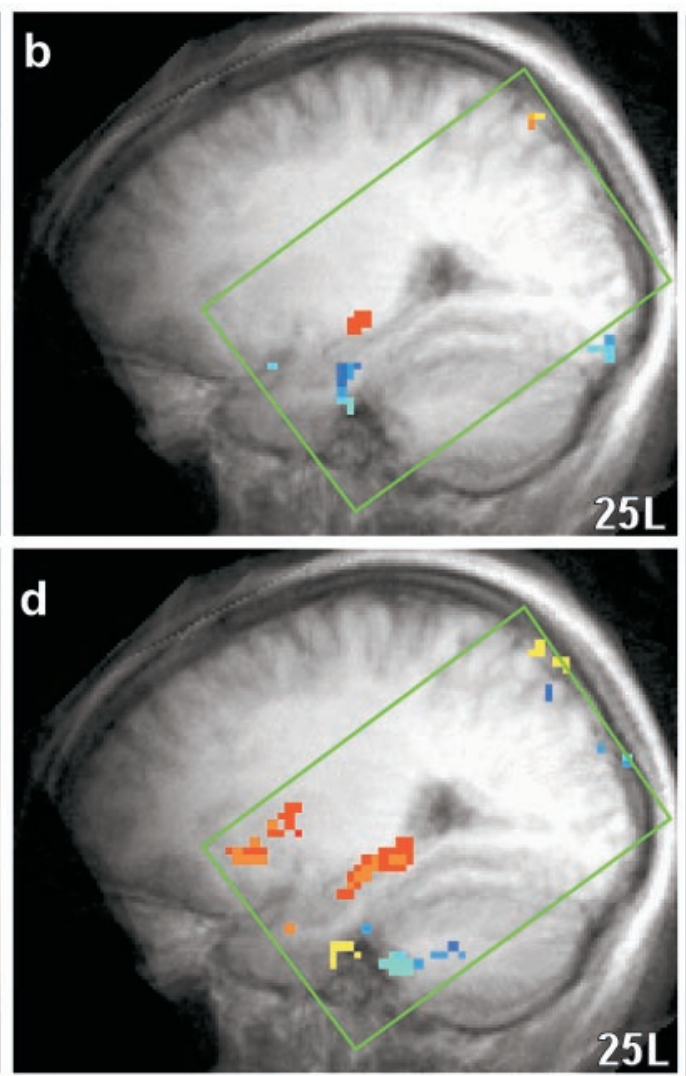

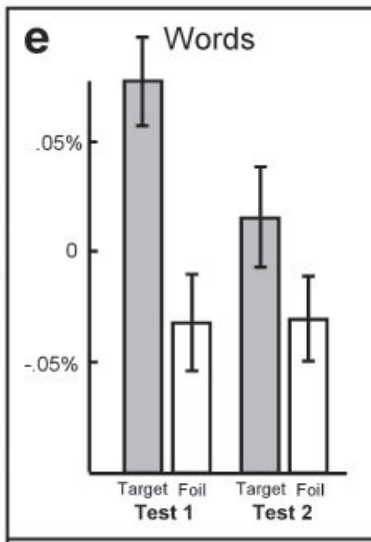

f Objects

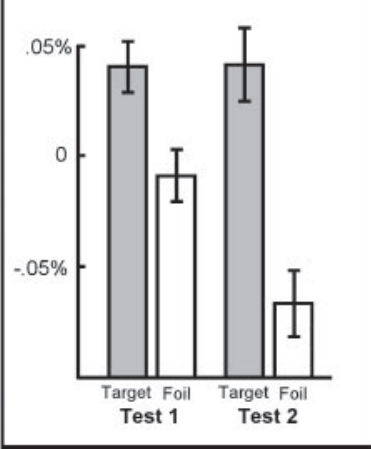

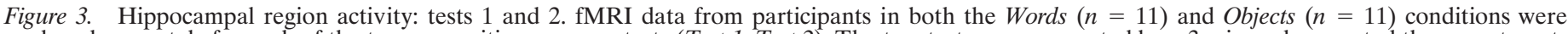

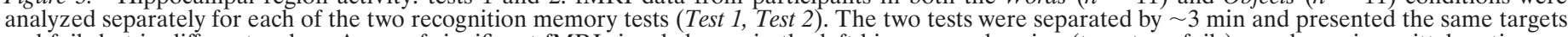

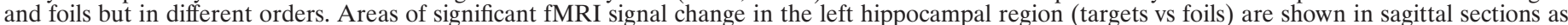

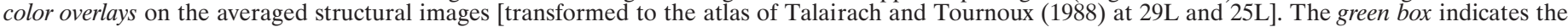

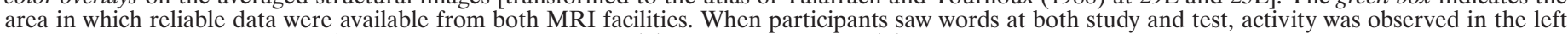

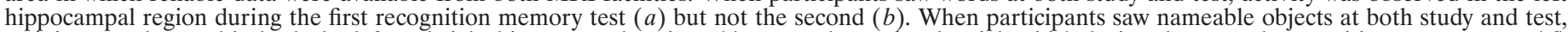

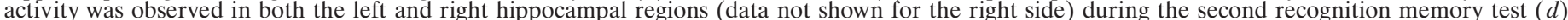

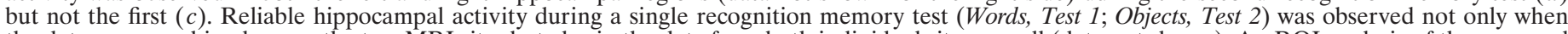

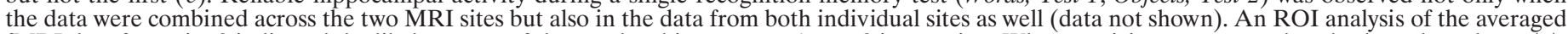

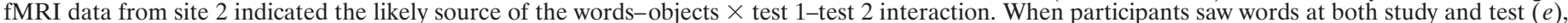

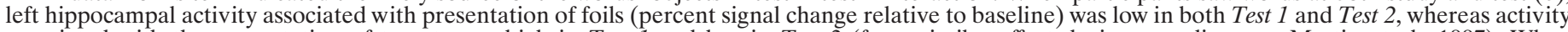

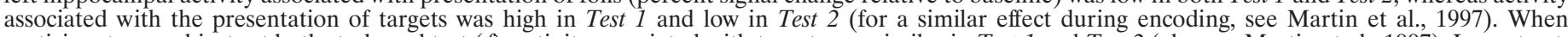

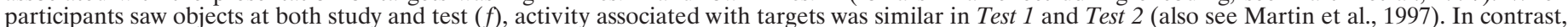

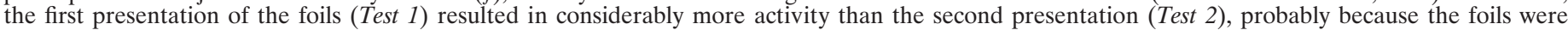

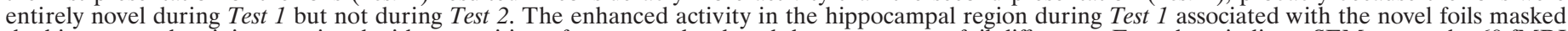

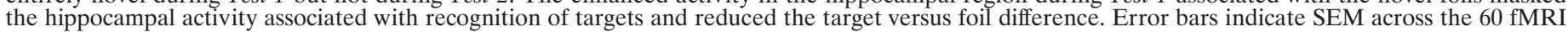
samples in the averaged data that were associated with presentation of either targets or foils.

at both study and test (Fig. 3e), the first recognition memory test revealed a large difference between targets and foils. This difference was much reduced during the second recognition memory test, because much less activity was associated with the targets. When participants saw objects at both study and test, the results were opposite (Fig. $3 f$ ). Only the second recognition test revealed a large difference between targets and foils, because during the first test the foils were associated with a relatively high level of activity.

\section{DISCUSSION}

Activation was observed in the hippocampal region during recognition memory testing when either words or pictures of nameable objects were used at both study and test. Activation was left unilateral for words and bilateral for objects, as has been reported previously in the case of memory encoding (Gabrieli et al., 1997; Martin et al., 1997; Wagner et al., 1997; Brewer et al., 1998; Kelly et al., 1998). This pattern of activation was reliable, because it was observed independently at two different MRI facilities. It should be noted that activity associated with recollective success was not consistently localized to either the more anterior or posterior region of the hippocampus (for discussion, see Gabrieli et al., 1997; Lepage et al., 1998; Schacter and Wagner, 1999).

There are at least two reasons why activity of the hippocampal region during retrieval, in association with recollective success, has not been commonly reported. First, the physical properties of the hippocampal region (its small size and proximity to the sinus cavity) make obtaining reliable data from the hippocampal region more difficult than from other cortical areas. Second, the difference in activity associated with targets and foils can often be reduced by activity associated with the encoding of foils. Several studies have found increased activity in the MTL associated with unfamiliar stimuli (relative to familiar stimuli), even when participants are instructed to view the stimuli passively (Stern et al., 1996; Lepage et al., 1998; Schacter and Wagner, 1999). This relatively automatic activation of the MTL associated with the encoding of unfamiliar stimuli works in opposition to finding a target versus foil contrast during recognition memory testing. Thus, the activity associated with the presentation of foils was high in test 1 (Fig. $3 f$ ), largely masking the difference in activity between targets and foils. In test 2 , when the foils were more familiar, the activity associated with 
foils decreased substantially, revealing a large difference in activity between targets and foils.

Interestingly, when words were used at both study and test (Fig. $3 e$ ), the activity associated with foils was low in both recognition tests. Presumably because of their high preexperimental familiarity, words have been shown to produce less activity in association with encoding than do objects (Kelly et al., 1998). In both tests 1 and 2, this factor may have contributed to the low level of activity for foil words. We also note that the activity associated with target words was lower in test 2 than in test 1 . We suggest that, for both words and objects, each time the test is administered and the targets and foils become more familiar, the activity associated with targets and the foils will become more similar. One might therefore expect that the level of activity in the hippocampal region associated with targets and foils would eventually converge. At the beginning of the present study, the words were more familiar than the pictures and therefore exhibited a reduction in target activity sooner than the pictures (i.e., from test 1 to test 2 ). It is possible that with more than two repetitions of the test, activity associated with target objects would also have diminished.

The experiments reported here used a variant of a blocked fMRI design in which the presentation of stimuli alternates between primarily targets and primarily foils. A purely blocked design represents one extreme of a fundamental tradeoff between optimizing trial-to-trial variability in stimulus presentation and the efficiency of the fMRI design (Friston et al., 1999). In a purely blocked design, the stimulus type to be presented on the next trial is easily predicted by the current trial. However, a blocked design exploits the additive nature of the BOLD response to multiple stimuli and produces a substantially larger percent signal change between blocks than the percent signal change between single events. Therefore, the efficiency of estimating the difference in activity associated with the two stimulus types can be several times higher in a blocked design than in an event-related design (Friston et al., 1999). By including foil items in the blocks of targets and by including target items in the blocks of foils, we achieved an efficient fMRI design (similar to the "dynamic stochastic" design of Friston et al., 1999) but also encouraged participants to respond on the basis of their memory for the item rather than on the basis of block structure.

To determine whether participants did respond primarily on the basis of memory (rather than expectation), we compared recognition accuracy for the minority items in each block (i.e., the two foil items in each block of primarily target items and the two target items in each block of primarily foil items) with recognition accuracy for the majority items in each block. On average, participants scored $86 \%$ correct on the majority items and $76 \%$ correct on the minority items. Thus, there was only a small difference in recognition accuracy for majority relative to minority items. Furthermore, the four participants who demonstrated the largest differences were all at site 1 (average difference at site 1,13\%); the average difference at site 2 was $6 \%$. Considering that the fMRI results at sites 1 and 2 were identical, it seems unlikely that the blocked nature of the task affected the results in any significant way.

The finding of robust hippocampal activation during retrieval of words or objects shows that such activation occurs in traditional recognition memory tasks and does not require tasks that are cross-modal, overtly associative, or explicitly spatial (for another potential example, see Henson et al., 1999). The data are consistent with lesion studies that have demonstrated impaired visual recognition memory after damage limited to the hippocampal region in humans and nonhuman primates (Reed and Squire, 1997; BeasonHeld et al., 1999; Zola et al., 2000). They are also consistent with single-unit data of cells within the hippocampus that indicate visual or olfactory recognition as an abstract match-nonmatch signal, which sometimes occurs in conjunction with other task features (Hampson et al., 1993, 1999; Fried et al., 1997; Wiebe and Staubli, 1999; Wood et al., 1999; Suzuki and Eichenbaum, 2000). Taken together, the available data indicate that the hippocampal region is involved rather broadly during recognition memory performance.
It is also worth noting that the current findings are wholly consistent with a role for the hippocampus in learning about relationships and conjunctions among stimuli (Sutherland and Rudy, 1989; Eichenbaum et al., 1994; Reed and Squire, 1999). In the recognition memory task studied here, the item presented during the retention test must be identified as what was presented during learning. Thus, at the time of learning, an association must be made between a to-be-remembered item and its context, and later this association must be retrieved. Indeed, the use of this kind of association can be considered an example of the process of rapidly forming conjunctions and retaining them across time that is fundamental to declarative memory. The present findings emphasize the fact that forming or retrieving an explicit association between two stimuli (e.g., an object and a word) is not required to engage the hippocampal region. It is sufficient simply to recognize the same stimulus that was presented at study. The question of how long after learning hippocampal activity remains important for retrieval (Milner et al., 1998; Bontempi et al., 1999; Stark and Squire, 2000) is an important topic for future studies.

\section{REFERENCES}

Aggleton JP, Brown MW (1999) Episodic memory, amnesia, and the hippocampal-anterior thalamic axis. Behav Brain Sci 22:425-444.

Beason-Held LL, Rosene DL, Killiany RJ, Moss MB (1999) Hippocampal formation lesions produce memory impairment in the rhesus monkey. Hippocampus 9:562-574.

Bontempi B, Laurent-Demir C, Destrade C, Jaffard R (1999) Timedependent reorganization of brain circuitry underlying long-term memory storage. Nature 400:671-675.

Brewer JB, Z hao Z, Glover GH, Gabrieli JDE (1998) Making memories: brain activity that predicts whether visual experiences will be remembered or forgotten. Science 281:1185-1187.

Buckner RL, Petersen SE, Ojemann JG, Miezin FM, Squire LR, Raichle ME (1995) Functional anatomical studies of explicit and implicit memory retrieval tasks. J Neurosci 15:12-29.

Collins DL, Neelin P, Peters TM, Evans AC (1994) Automatic 3D intersubject registration of MR volumetric data in standardized Talairach space. J Comp Assist Tomogr 18:192-205.

Cox RW (1996) AFNI: Software for analysis and visualization of functional magnetic resonance neuroimages. Comp Biomed Res 29:162-173.

Eichenbaum H, Otto T, Cohen NJ (1994) Two functional components of the hippocampal memory system. Behav Brain Sci 17:449-518.

Fernandez G, Weyerts H, Schrader-Bolsche M, Tendolkar I, Smid HG, Tempelmann C, Hinrichs H, Scheich H, Elger CE, Mangun GR, Heinze HJ (1998) Successful verbal encoding into episodic memory engages the posterior hippocampus: a parametrically analyzed functional magnetic resonance imaging study. J Neurosci 18:1841-1847.

Fletcher PC, Frith CD, Grasby PM, Shallice T, Frackowiak RS, Dolan RJ (1995) Brain systems for encoding and retrieval of auditory-verbal memory. An in vivo study in humans. Brain 118:401-416.

Fried I, MacDonald KA, Wilson CL (1997) Single neuron activity in human hippocampus and amygdala during recognition of faces and objects. Neuron 18:753-765.

Friston KJ, Zarahn E, Josephs O, Henson RN, Dale AM (1999) Stochastic designs in event-related fMRI. Neuroimage 10:607-619.

Fujii T, Okuda J, Kawashima R, Yamadori A, Fukatsu R, Suzuki K, Ito M, Goto R, Fukuda H (1997) Different roles of the left and right parahippocampal regions in verbal recognition: a PET study. NeuroReport 8:1113-1117.

Gabrieli JDE, Brewer JB, Desmond JE, Glover GH (1997) Separate neural bases of two fundamental memory processes in the human medial temporal lobe. Science 11:264-266.

Grasby PM, Frith CD, Friston KJ, Bench C, Frackowiak RS, Dolan RJ (1993) Functional mapping of brain areas implicated in auditory-verbal memory function. Brain 116:1-20.

Hampson RE, Heyser CJ, Deadwyler SA (1993) Hippocampal cell firing correlates of delayed-matching-to-sample performance in the rat. Behav Neurosci 107:715-739.

Hampson RE, Simeral JD, Deadwyler SA (1999) Distribution of spatial and nonspatial information in dorsal hippocampus. Nature 402:610-614.

Haxby JV, Ungerleider LG, Horwitz B, Maisog JM, Rapoport SI, Grady CL (1996) Face encoding and recognition in the human brain. Proc Natl Acad Sci USA 93:922-927.

Henke K, Buck A, Weber B, Wieser HG (1997) Human hippocampus establishes associations in memory. Hippocampus 7:249-256.

Henke K, Weber DA, Kneifel L, Wieser HG, Buck A (1999) Human hippocampus associates information in memory. Proc Natl Acad Sci USA 96:5884-5889.

Henson RNA, Rugg MD, Shallice T, Josephs O, Dolan RJ (1999) Recollection and familiarity in recognition memory: an event-related functional magnetic resonance imaging study. J Neurosci 19:3962-3972.

Kapur S, Craik FI, Tulving E, Wilson AA, Houle S, Brown GM (1994) 
Neuroanatomical correlates of encoding in episodic memory: levels of processing effect. Proc Natl Acad Sci USA 91:2008-2011.

Kelly W, Miezin F, McDermott K, Buckner RL, Raichel ME, Cohen NJ, Ollinger JM, Akbudak E, Contura TE, Snyder AZ, Peterson SE (1998) Hemispheric specialization in human dorsal frontal cortex and medial temporal lobe for verbal and nonverbal memory encoding. Neuron 20:927-936.

Lepage M, Habib R, Tulving E (1998) Hippocampal PET activations of memory encoding and retrieval: the HIPER model. Hippocampus $8: 313-322$.

Martin A, Wiggs CL, Weisberg J (1997) Modulation of human medialtemporal lobe activity by form, meaning, and experience. Hippocampus 7:587-593.

Milner B, Squire LR, Kandel ER (1998) Cognitive neuroscience and the study of memory. Neuron 20:445-468.

Murray EA, Mishkin M (1998) Object recognition and location memory in monkeys with excitotoxic lesions of the amygdala and hippocampus. J Neurosci 18:6568-6582.

Nyberg L, Tulving E, Habib R, Nilsson S, Kapur S, Houle S, Cabeza R, McIntosh AR (1995) Functional brainmaps of retrieval mode and recovery of episodic information. NeuroReport 7:249-252.

Reber PJ, Wong EC, Buxton RB, Frank LR (1998) Correction of offresonance related distortion in EPI using EPI based field maps. Magn Reson Med 39:328-330.

Reed JM, Squire LR (1997) Impaired recognition memory in patients with lesions limited to the hippocampal formation. Behav Neurosci 111:667-675.

Reed JM, Squire LR (1999) Impaired transverse patterning in human amnesia is a special case of impaired memory for two-choice discrimination tasks. Behav Neurosci 113:3-9.

Schacter DL, Wagner AD (1999) Medial temporal lobe activations in fMRI and PET studies of episodic encoding and retrieval. Hippocampus 9:7-24.

Schacter DL, Buckner RL, Koutstaal W, Dale AM, Rosen BR (1997) Late onset of anterior prefrontal activity during retrieval of veridical and illusory memories: a single trial FMRI study. Neuroimage 6:259-269.

Shallice T, Fletcher P, Frith CD, Grasby P, Frackowiak RS, Dolan RJ (1994) Brain regions associated with acquisition and retrieval of verbal episodic memory. Nature 368:633-635.
Snodgrass JG, Vanderwart M (1980) A standardized set of 260 pictures: norms for name agreement, image agreement, familiarity, and visual complexity. J Exp Psychol Hum Learn Mem 6:174-215.

Stark CEL, Squire LR (2000) fMRI activity in the medial temporal lobe during recognition memory as a function of study-test interval. Hippocampus $10: 329-337$.

Stern CE, Corkin S, Gonzalez RG, Guimaraes AR, Baker JR, Jennings PJ, Carr CA, Sugiura RM, Vadantham V, Rosen BR (1996) The hippocampal formation participates in novel picture encoding: evidence from functional magnetic resonance imaging. Proc Natl Acad Sci USA 93:8600-8665.

Sutherland RW, Rudy JW (1989) Configural association theory: the role of the hippocampal formation in learning, memory and amnesia. Psychobiology 17:129-144.

Suzuki WA, Eichenbaum H (2000) The neurophysiology of memory. Ann NY Acad Sci 911:175-191.

Talairach J, Tournoux P (1988) A co-planar stereotaxic atlas of the human brain. New York: Thieme.

Tulving E, Kapur S, Markowitsch HJ, Craik FI, Habib R, Houle S (1994) Neuroanatomical correlates of retrieval in episodic memory: auditory sentence recognition. Proc Natl Acad Sci USA 91:2012-2015.

Wagner AD, Gabrieli JDE, Verfallie M (1997) Building memories: Remembering and forgetting of verbal experiences as predicted by brain activity. Science 281:1188-1191.

Wiebe SP, Staubli UV (1999) Dynamic filtering of recognition memory codes in the hippocampus. J Neurosci 19:10562-10574.

Wong EC, Bandettini PA, Hyde JS (1992a) Echoplanar imaging of the human brain using a three axis local gradient coil. In: Proceedings of the Society of Magnetic Resonance in Medicine 11th Annual Meeting, p 105. Berlin: Society of Magnetic Resonance in Medicine.

Wong EC, Boskamp E, Hyde JS (1992b) A volume optimized quadrature elliptical endcap birdcage brain coil. In: Proceedings of the Society of Magnetic Resonance in Medicine 11th Annual Meeting, p 4015. Berlin: Society of Magnetic Resonance in Medicine.

Wood ER, Dudchenko PA, Eichenbaum H (1999) The global record of memory in hippocampal neuronal activity. Nature 397:613-616.

Zola SM, Squire LR, Teng E, Stefanacci L, Buffalo EA, Clark RE (2000) Impaired recognition memory in monkeys after damage limited to the hippocampal region. J Neurosci 20:451-463. 